\title{
Role of mental health professionals in the management of functional somatic symptoms in primary care ${ }^{\dagger}$
}

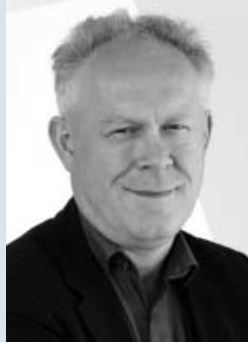

Richard Morriss

\section{Summary}

Functional somatic symptoms associated with persistent frequent attendance is emotionally demanding, costly and intractable to treat. Such patients are hard to engage in practice and research by mental health professionals, whose main role may be indirect training, supporting and advising primary care professionals rather than direct patient care.

\section{Declaration of interest}

None.
Richard Morriss is Professor of Psychiatry and Community Mental Health at Nottingham University and Director of Research for the National Institute for Health Research Collaboration for Leadership in Applied Health Research and Care (NIHR CLAHRC) for Nottinghamshire, Derbyshire and Lincolnshire.

Functional somatic symptoms or medically unexplained physical symptoms are associated with distress and/or functional impairment but are not explained by organic physical disease. Functional somatic symptoms are found in up to a third of those seeking help with physical symptoms in primary care. ${ }^{1}$ Given that the long-term care of these individuals is usually the responsibility of primary care, what do we know from research about the nature and role of the mental health professional in supporting primary care professionals in this task?

\section{Diagnostic uncertainty}

Unlike specialist secondary care settings, clinical practice in highvolume primary care precludes diagnostic certainty that there is no underlying organic disease. Comprehensive investigation of every patient with functional somatic symptoms would be prohibitively expensive and impractical. Instead, general practitioners and health professionals in these settings make clinical judgements about the most likely explanation for physical symptoms, relying on 'red flag' symptoms and signs such as unexplained bleeding and weight loss, focused examination and investigation and the progression of symptoms to indicate clinically important underlying pathology. Given that up to $10 \%$ of symptoms initially judged by general practitioners to be functional somatic symptoms will manifest later to have been the first presentation of organic disease, ${ }^{2}$ functional somatic symptoms are best considered as a working hypothesis based on probability rather than a definitive diagnosis. Both the patient and the doctor understand this and rely on the fact that symptoms will progress if organic physical disease was missed at first consultation. ${ }^{3}$ Therefore in primary care settings, interventions that imply certainty about the non-organic nature of functional somatic symptoms such as reattribution are not always convincing to patients, who may worry that future presentations of physical symptoms to that doctor might not be given due consideration in terms of the possibility of an underlying serious physical health condition. ${ }^{3}$

†See pp. 499-507, this issue.

\section{Persistent frequent attendance}

Many patients with less severe functional somatic symptoms are managed well by health professionals in primary care settings. ${ }^{4}$ The outcomes and frequency of consultation of patients with such symptoms are similar to those of patients with medically explained symptoms in primary care settings and mental disorders with psychologising attributions for their symptoms. ${ }^{5}$ Increased cost and poorer outcomes emerge only when patients have multiple functional somatic symptoms, or have symptoms that are complicated by depression and/or anxiety disorder or frequent attendance. When asked about functional somatic symptoms, primary care professionals are most concerned about patients who also have frequent attendance as they are the most emotionally demanding and expensive to treat. However, six out of seven patients who are in the top $10 \%$ of frequent attendance in primary care, adjusted for age and gender, return to normal patterns of healthcare attendance within 12 months. On the other hand, frequent attendance persisting for 2 years may become an enduring problem, with less than $2 \%$ of the general practitioner's list taking up $11 \%$ of all available primary care consultations, ${ }^{6}$ with greater than average secondary healthcare consumption. Help with this group of patients might both reduce health costs and improve limited access to care for other patients facing delays in receiving the healthcare that they require.

However, patients with functional somatic symptoms and persistent frequent attendance are hard to engage in clinical practice or psychological treatment trials despite their proclivity for high usage of healthcare. Such patients may believe terms such as 'mental', 'psychological' or even 'frequent attender' indicate that health professionals disbelieve their symptoms, think that the patient has made them up or view them as weak or selfish individuals who are to blame for their symptoms. ${ }^{7}$ As a result, patients with functional somatic symptoms who have relatively normal patterns of consultation and less negative views concerning mental health are often enrolled in psychological treatment trials and the results may not be generalisable to patients who are harder to engage. Recently published randomised controlled trials of moderately effective psychological interventions have included some patients who probably do have both functional somatic symptoms and persistent frequent attendance, ${ }^{8}$ but the sample size, setting and design of the study preclude any definitive statement about the effectiveness of these approaches and other interventions such as individual cognitive-behavioural therapy in the persistently frequent attending group with functional somatic symptoms. 


\section{Role of the psychiatrist}

The evidence base for effective interventions for functional somatic symptoms associated with frequent attendance in primary care is extremely thin, with few well-conducted, large randomised controlled trials. ${ }^{1}$ The complexity of these patients' developmental, physical, health, mental health, iatrogenic and social problems would suggest that a psychiatrist, rather than a non-medically qualified mental health professional familiar with the general healthcare context, would be ideally placed to help primary care professionals with the longer-term management of these patients. The growing evidence base for the effectiveness of interventions such as cognitive-behavioural therapy, ${ }^{9}$ and the need sometimes for multi-agency social care, suggest roles for other non-medically qualified mental health professionals as well as the psychiatrist.

Research has shown some benefits of psychiatrists taking such a role through stepped care, joint consultation and consultation letter approaches. ${ }^{1}$ These interventions rely on the organisation of care so that patients see the same general healthcare professional on a regular but gradually diminishing basis with relatively little face-to-face care with the psychiatrist. In between appointments with their usual health professional, these patients are encouraged by reception and triage staff to wait to have contact with their usual general healthcare professional rather than to see another healthcare professional who is less familiar with their condition. Involvement of the patient in direct consultation with secondary care professionals is discouraged unless there is a clear and compelling health reason. Fortunately, most patients prefer such an approach provided the contact with their usual general health professional is timely enough for patients to be able to contain their health anxiety and there is an absence of 'red flag' signs of the need for more urgent physical or mental healthcare, such as suicidal plans associated with depression.

Close attention is paid to establishing an empathic relationship with consistent communication, and the general healthcare professional tries to establish a non-blaming alliance with the patient to improve his or her health. Any episodes of catastrophic misinterpretation of physical symptoms are explored with the patient and used as an opportunity for educating the patient. All the physical, mental and lifestyle health concerns that the patient and doctor have at the outset are addressed through history taking, examination, investigation and explanation. Only new symptoms or progression of existing symptoms are investigated at later consultations, thereby addressing the patient's and doctor's concerns that physical disease will not be missed.

Opportunities to promote self-management, adaptive coping and health promotion are encouraged. The combination of improving self-efficacy in relation to health and reducing threat from health through education, and openness to the possibility of reconsideration of health problems should they become worse, has the potential to reduce health anxiety and the need for consultation with health professionals to seek reassurance. Medication approaches may include not only antidepressants to treat depression and anxiety but also the rationalisation of counterproductive medication strategies such as excessive sedation from opiates used for unexplained pain or salbutamol for difficulties with breathing due to anxiety wrongly attributed to asthma. Social, employment and family problems that might increase anxiety or depression are also addressed. Such an approach to care is strategic and has to be applied over many months, requiring continuing mental health professional support, in contrast to most work in primary care settings that is reactive to the demands of the patient or involves simple proactive health monitoring.

\section{Workforce and the need for further research}

The role of a psychiatrist is largely in training and emotionally supporting the primary care professional, as well as a limited amount of direct patient care helping the primary care professional to make an assessment and a management plan. Direct involvement of a mental health professional will be required if the patient develops serious mental health complications such as high suicide risk, or if over time the patient develops sufficient acceptance and understanding of the underlying psychosocial problems to benefit from a more formal course of psychological treatment. Some countries are better placed than others to provide this kind of mental health professional support to general healthcare because they have aworkforce trained to assess and manage functional somatic symptoms (e.g. Germany) or a tradition of supervision by mental health professionals in primary care and a payment system to reward such work, as in some parts of Spain. Unfortunately, in the UK, mental health professionals have been infrequently trained for such a role and the skills of liaison psychiatry are confined to the general hospital, primary care professionals tend to work individually without supervision from mental health professionals, and the tariff system does not reward primary care for work with patients with functional somatic symptoms or mental health professionals to work with primary care without seeing the patient on a face-toface basis. Research is required now to determine whether the liaison approach by mental health professionals to general health professionals outlined here is both clinically effective and cost-effective, to estimate accurately the potentially huge health costs of care of this relatively small group of patients, and to explore how the competencies of the current workforce, organisation of care and tariff system might be improved or reformed.

\section{Acknowledgement}

R.M. is funded by NIHR for researching frequent attendance in primary care. The views expressed are those of the author and not necessarily those of the NHS, NIHR or Department of Health.

Richard Morriss, FRCPsych, Department of Psychiatry and Community Mental Health, Institute of Mental Health, Floor B, Sir Colin Campbell Building, University of Nottingham Innovation Park, Triumph Road, Nottingham NG7 2TU, UK. E-mail: richard.morriss@nottingham.ac.uk

First received 7 Oct 2011, final revision 22 Dec 2011, accepted 17 Jan 2012

\section{References}

1 Hoedeman R, Blankenstein AH, van der Feltz-Cornelis CM, Krol B, Stewart R, Groothoff JW. Consultation letters for medically unexplained physical symptoms in primary care. Cochrane Database Syst Rev 2010; 12: CD006524.

2 Gask L, Dowrick C, Salmon P, Peters S, Morriss R. Reattribution reconsidered: reflections on an educational intervention for medically unexplained symptoms in primary care settings. J Psychosom Res 2011; 71: 325-34.

3 Peters S, Rogers A, Salmon P, Gask L, Dowrick C, Towey M, et al. What do patients choose to tell their doctors? Qualitative analysis of potential barriers to reattributing medically unexplained symptoms. J Gen Intern Med 2009; 24: 443-9.

4 Salmon $\mathrm{P}$, Peters S, Clifford R, Iredale W, Gask L, Rogers A, et al. Why do general practitioners decline training to improve management of medically unexplained symptoms? J Gen Intern Med 2007; 22: 565-71.

5 Kisely S, Simon G. An international study comparing the effect of medically explained and unexplained somatic symptoms on psychosocial outcome. J Psychosom Res 2006; 60: 125-30.

6 Smits FT, Brouwer HJ, van Weert HC, Schene AH, ter Riet G. Predictability of persistent frequent attendance: a historic 3-year cohort study. Br J Gen Pract 2009; 59: e44-50.

7 Salmon P, Peters S, Stanley I. Patients' perceptions of medical explanations for somatisation disorders: qualitative analysis. BMJ 1999; 318: 372-6.

8 Schröder A, Rehfeld E, Ørnbøl E, Sharpe M, Licht RW, Fink P. Cognitivebehavioural group treatment for a range of functional somatic syndromes: randomised trial. Br J Psychiatry 2012; 200: 499-507.

9 Escobar Jl, Gara MA, Diaz-Martinez AM, Interian A, Warman M, Allen LA, et al. Effectiveness of a time-limited cognitive behavior therapy type intervention among primary care patients with medically unexplained symptoms. Ann Fam Med 2007; 5: 328-35. 